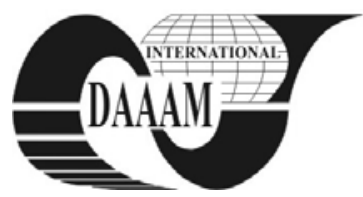

Annals of DAAAM for 2011 \& Proceedings of the 22nd International DAAAM Symposium, Volume 22, No. 1, ISSN 1726-9679 ISBN 978-3-901509-83-4, Editor B. Katalinic, Published by DAAAM International, Vienna, Austria, EU, 2011 Make Harmony between Technology and Nature, and Your Mind will Fly Free as a Bird Annals \& Proceedings of DAAAM International 2011

\title{
USE OF IMPROVED ITERATIVE CORRECTION OF RADIAL DISTORTION
}

\author{
RAVAS, R[udolf] \& SYROVA, L[ivia]
}

\begin{abstract}
The paper treats the topic of estimation of coefficients and the centre of radial distortion of the image. New method of coefficients and coordinates of the centre of radial distortion estimation of the image is presented. Method is based on the iterative correction of coordinates of selected points of the distorted straight lines. It does not require a parametric expression of distorted lines. Analysis of selected properties of method by simulated and real images is presented as well.

Key words: radial distortion, centre of distortion, image correction, straight line
\end{abstract}

\section{INTRODUCTION}

During measurement of spatial coordinates of scene interest points by non-metric cameras it is necessary to establish camera model parameters. Model is established by intrinsic and extrinsic camera parameters. Intrinsic parameters describe relationship between position of the point in the space with coordinates expressed in the coordinate system of the camera and position of the image of the same point expressed in the image coordinate system (Devernay \& Faugeras, 2001). Model describes the mechanism of distortion of image point position caused by the imperfection of camera lens as well. The now widely recognized significant source of distortion caused by the camera optics is radial distortion of the image (Fakhrul, 2009) described by model (Bräuer-Burchardt, 2004)

$$
r_{d}=r\left(1+\alpha_{2} r^{2}+\alpha_{4} r^{4}+\cdots\right)
$$

where $\mathrm{r}$ is distance of the point in ideal undistorted image to the centre of distortion $C=\left(c_{d x}, c_{d y}\right), r_{d}$ is the distance of the point in the radial distorted image to the centre of distortion, $\alpha_{\mathrm{i}}$ are coefficients of radial distortion. The frequently used models are polynomials of the second, maximal the fourth order (BräuerBurchardt, 2004). Existing methods of radial distortion correction can be divided into two groups, the first one (multiple view method) is based on the use of multiple view the second approach is characterized by the use of only single image (Wang et al., 2009). Methods using one image usually utilize the fact that at the perspective projection (assumption in many models) the ideal image of the straight line in space is straight line. It is necessary to detect images of the potential straight lines. Models of the distorted images of are quadratic curves well approximating model (1). The individual methods differentiate by the mode of approximation of images of line, by the number of parameters and by the number of parameters of model and whether the centre of radial distortion would be calculated or whether centre of radial distortion with the principal point of the image would be identified (Bouguet, 2010). Method presented in this paper enables to calculate the centre of distortion what is emphasized in many papers (Wang et al., 2009), (Hartley \& Kang, 2007). It represents modification of the method (Ravas et al., 2011) increasing stability and accuracy of calculating the coordinates of the centre and parameters of radial distortion. Method does not require to parametrize distorted image of the straight line.

\section{SUBJECT AND METHOD}

Let's suppose to have $K$ sets of images $p_{k i}$ of selected points with coordinates $\left(\mathrm{x}_{\mathrm{ki}}, \mathrm{y}_{\mathrm{ki}}\right)$ that are in the distorted image. Images of $p_{k i}$ should lie on the straight line $L_{k}$ in ideal undistorted image. Model (1) is replaced by models considering unequal distortion along the coordinate axes of the image that can be for each point $\mathrm{p}_{\mathrm{ki}}$ expressed

$r_{x k i}=\frac{r_{d k i}}{\left(1+\beta_{x 2} q_{d k i}^{2}+\beta_{x 4} q_{d k i}^{4}+\cdots\right)} \quad r_{y k i}=\frac{r_{d k i}}{\left(1+\beta_{y 2} q_{d k i}^{2}+\beta_{y 4} q_{d k i}^{4}+\cdots\right)}$

where $r_{x k i}, r_{y k i}$ are relative coordinates of point $p_{k i}$ to the centre $C=\left(c_{d x}, c_{d y}\right)$ of radial distortion in undistorted image, $r_{d k i}$ is the distance of $\mathrm{p}_{\mathrm{ki}}$ to the centre of radial distortion in distorted image, $\beta_{\mathrm{xi}}, \beta_{\mathrm{yj}}$, are coefficients of the inverse radial distortion along axes $\mathrm{x}, \mathrm{y}, \mathrm{q}_{\mathrm{dki}}=\mathrm{r}_{\mathrm{dki}} / \mathrm{D}$ is normalized distance with suitable chosen constant $\mathrm{D}$. Coordinates $\left(\mathrm{x}_{\mathrm{cki}}, \mathrm{y}_{\mathrm{cki}}\right)$ of points $\mathrm{p}_{\mathrm{cki}}$ in the undistorted image corresponding to the points $\mathrm{p}_{\mathrm{ki}}$ can be expressed by

$$
x_{k i c}=r_{x k i}+c_{d x} \quad y_{k i c}=r_{y k i}+c_{d y}
$$

As the equation of the straight line $L_{k}$ isn't known we use straight line $\mathrm{l}_{\mathrm{k}}(\mathrm{x}, \mathrm{y})$ that approximates all points $\mathrm{p}_{\mathrm{cki}}$ by LSQ method

$$
l_{k}(x, y)=a_{k} x+b_{k} y+c_{k}=0 \sqrt{a_{k}^{2}+b_{k}^{2}}=1
$$

Square of perpendicular distance of point $\mathrm{p}_{\mathrm{cki}}$ to the straight line (4) will be

$$
d_{c k i}^{2}=l_{k}^{2}\left(x_{c k i}, y_{c k i}\right)=\left(a_{k} x_{c k i}+b_{k} y_{c k i}+c_{k}\right)^{2}
$$

All corrected points lie on the straight lines (4). We know neither corrected coordinates (3) at the start of solution nor equations of approximating lines (4). Therefore correction of radial distortion of the image is based on the iterative estimation of values of unknown parameters such as the centre of radial distortion $\mathrm{C}=\left(\mathrm{c}_{\mathrm{dx}}, \mathrm{C}_{\mathrm{dy}}\right)$ and coefficients from (2). As the objective is to minimize cost function of parameters $\omega=\left[\mathrm{c}_{\mathrm{dx}}, \mathrm{c}_{\mathrm{dy}}, \beta_{\mathrm{x} 2}, \beta_{\mathrm{x} 4}, \ldots, \beta_{\mathrm{y} 2}, \beta_{\mathrm{y} 4}, \ldots\right]^{\mathrm{T}}$ defined by

$$
J(\omega)=\min _{\omega} \sum_{k=1}^{K} \sum_{i=1}^{n k}\left(l_{k}\left(x_{k i c}, y_{k i c}\right)\right)^{2}
$$

where $\mathrm{n}_{\mathrm{k}}$ is number of points corresponding to the $\mathrm{k}$-th line.

\section{RESULTS}

In order to analyze properties of the proposed method simulation experiments and real images of chessboard captured by CCD camera have been used. In both experiments ideal images of points arranged in a grid were examined. Images of grid points in ideal case lie on related vertical and horizontal 
lines. Criteria published in (Bräuer-Burchardt, 2004) have been used to quantify successfulness of correction. Criteria such ARE and MRE for deviation of corrected distorted points $\mathrm{p}_{\mathrm{ic}}=\left(\mathrm{x}_{\mathrm{ic}}, \mathrm{y}_{\mathrm{ic}}\right)$ to ideal image ones $\mathrm{p}_{\mathrm{iI}}=\left(\mathrm{x}_{\mathrm{Ii}}, \mathrm{y}_{\mathrm{Ii}}\right)$ are defined by

$$
A R E=\frac{1}{N} \sum_{i=1}^{N}\left\|p_{i c}-p_{i I}\right\|_{2} \quad M R E=\max _{i}\left\|p_{i c}-p_{i I}\right\|_{2}
$$

Criteria corresponding to the deviation of corrected points from approximating straight lines $d_{\text {cki }}(5)$ that are average value (AREL) and standard deviation (MREL) of $\mathrm{d}_{\mathrm{cki}}$ are defined by

$$
A R E L=\frac{1}{\mathrm{~K}} \sum_{k=1}^{K} \frac{1}{n k} \sum_{i=1}^{n k} \sqrt{d_{c k i}^{2}} \quad M R E L=\max _{i, k} \sqrt{d_{c k i}^{2}}
$$

As important information we can consider that the use of suitable chosen normalization factor enabling ensures stability of calculation of radial distortion parameters.

\begin{tabular}{|l|l|l|l|l|}
\hline $\mathrm{D}$ & ARE & MRE & AREL & MREL \\
\hline 2000 & 0.0040 & 0.0083 & $3.026 \mathrm{e}-005$ & $3.316 \mathrm{e}-004$ \\
\hline 1500 & 0.0040 & 0.0072 & $2.620 \mathrm{e}-005$ & $2.744 \mathrm{e}-004$ \\
\hline 1000 & 0.0029 & 0.0066 & $2.751 \mathrm{e}-005$ & $2.782 \mathrm{e}-004$ \\
\hline 900 & 0.0058 & 0.0095 & $8.447 \mathrm{e}-005$ & $7.691 \mathrm{e}-004$ \\
\hline 800 & 0.5068 & 1.1004 & 0.0060 & 0.0653 \\
\hline 500 & 1.2572 & 2.7792 & 0.0144 & 0.1509 \\
\hline 300 & 2.3465 & 4.2533 & 0.0254 & 0.1808 \\
\hline 100 & 324.21 & 569.68 & 7.0596 & 92.7197 \\
\hline
\end{tabular}

Tab. 1. Values ARE, MRE AREL a MREL depending upon selected values $\mathrm{D}$ for the simulated image distortion

In table 1 are values of ARE, MRE, AREL and MREL for simulated distorted image with points arranged into $5 \times 5$ grid according to the value $\mathrm{D}$. The table 1 shows that the value $\mathrm{D}$ affects not only the stability of calculation of distortion parameters (at the value 100 calculation diverges) but it affects accuracy of the correction as well (the most precise values are obtained for $\mathrm{D}=1000)$.

\begin{tabular}{|l|l|l|l|l|}
\hline Order & ARE & MRE & AREL & MREL \\
\hline 2 & 0.3361 & 0.6846 & 0.0052 & 0.0509 \\
\hline 4 & 0.0296 & 0.0605 & $2.764 \mathrm{e}-004$ & 0.0021 \\
\hline 6 & 0.0041 & 0.0084 & $3.014 \mathrm{e}-005$ & $3.294 \mathrm{e}-004$ \\
\hline 8 & 0.0032 & 0.0062 & $2.513 \mathrm{e}-005$ & $2.767 \mathrm{e}-004$ \\
\hline 10 & 0.0029 & 0.0066 & $2.751 \mathrm{e}-005$ & $2.782 \mathrm{e}-004$ \\
\hline
\end{tabular}

Tab. 2.Values ARE, MRE AREL and MREL depending upon selected values of the order of approximating polynomial for the simulated image distortion

In table 2 are values of ARE, MRE, AREL and MREL for the same simulated distorted image depending on the polynomial order in denominator of model (2) for the value $\mathrm{D}=1000$.

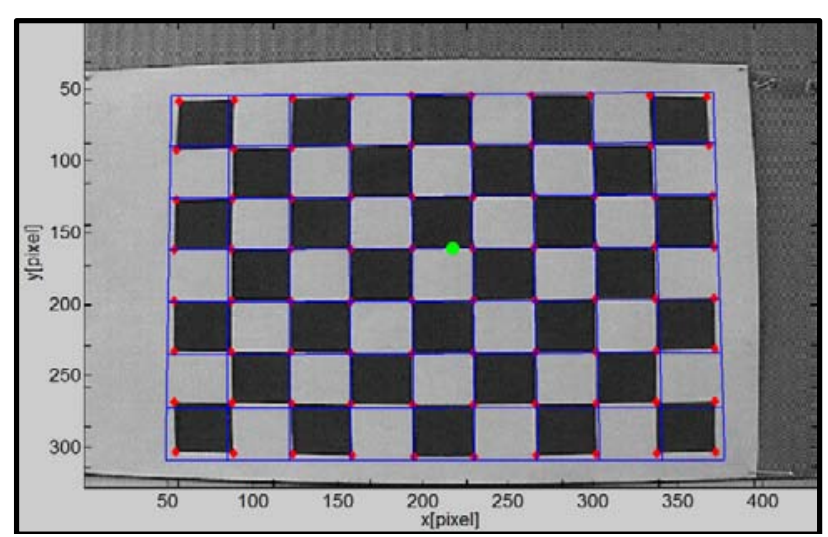

Fig. 1. Distorted image of the chessboard calibration object
Modeling of distortion was accomplished by polynomial of the $4^{\text {th }}$ order. Method allows to approximate radial distortion also by polynomials of higher order what can reduce error of correction of radial distortion.Images captured with camera (fig.1) have been used in experiments with real images. In ideal case there are corner points of chessboard as elements of vertical and horizontal straight lines on the chessboard. Corner points were extracted by Harris corner detector that can calculate coordinates of corner points with sub-pixel resolution. Positions of extracted points are in the image distinguished by the symbol "diamond". Position of the radial distortion is marked by the symbol "circle". The contours of corrected image of the chessboard are marked by lines. In this case we have used $\mathrm{D}=1000$ and the order of approximating polynomial was 4.

\section{CONCLUSION}

Experiments, some of which were presented in this paper, showed good properties of proposed method of correction of errors due to the image radial distortion. Variability of the choice of initial value of the distortion centre and of standardization constant didn't influence the convergence of solution. Initial values of radial distortion coefficients were always set to zero. Criteria AREL and MREL appear to be suitable for assessing the quality of correction in case that coordinates of points in ideal undistorted image are unknown. Maximum deviation to the approximating straight line during the correction of radial distortion of real image is less than 1 pixel. During the correction it is not necessary to know intristic parameters of camera. In future possibilities of the use of method will be examined at the calibration of camera intristic parameters.

\section{ACKNOWLEDGEMENTS}

We gratefully acknowledge that the results presented here were solved as partial goals of the research task VEGA No. 1/0490/10.

\section{REFERENCES}

Bouguet, J.Y. (2010). Camera Calibration Toolbox for Matlab®, Available from: http://www.vision.caltech.edu/ bouguetj/calib_doc/ Accessed on: 2010-10-10

Bräuer-Burchardt, Ch. (2004). A simple new method for precise lens distortion correction of low cost camera cystems, In: DAGM , C.E. Rasmussen et al. (Eds.), 570577, LNCS 3175

Devernay, F. \& Faugeras, O. (2001). Straight lines have to be straight. Machine Vision and Applications, Vol. 13, pp. 1424,

Fakhrul, Y., et al. (2009), Establishing the straightness of a line for radial distortion correction through conic fitting. IJCSNS International Journal of Computer Science and Network Security, Vol. 9, No. 5, (May 2009) pp. 286 - 293

Hartley, R. \& Kang, S.B. (2007). Parameter-Free Radial Distortion Correction with Center of Distortion Estimation. IEEE Transactions on Pattern Analysis and Machine Intelligence, Vol. 29, No. 8, (August 2007) pp. 1309-1321

Ravas, R.; Syrova, L. \& Grman, J. (2011). The iterative method of parameters and centre of radial distortion estimation, Proceedings of the $8^{\text {th }}$ International Conference, Smolenice, ISBN 978-80-969-672-4-7, Institute of Measurement Science SAS, Bratislava, Slovakia

Wang, A.; Qiu, T. \& Shao, L. (2009). A simple method of radial distortion correction with centre of distortion estimation. J Math Imaging Vis, Vol. 35, pp. 165-172 